Communication, technologies et développement

5 | 2018

Le numérique et le développement des Suds

\title{
Frédéric Antoine (dir.), Analyser la radio. Méthodes et pratiques
}

Idé Hamani

\section{OpenEdition}

Édition électronique

URL : http://journals.openedition.org/ctd/426

DOI : $10.4000 /$ ctd. 426

ISSN : 2491-1437

Éditeur

Chaire Unesco Pratiques émergentes en technologies et communication pour le développement

Référence électronique

Idé Hamani, « Frédéric Antoine (dir.), Analyser la radio. Méthodes et pratiques », Communication, technologies et développement [En ligne], 5 | 2018, mis en ligne le 02 janvier 2018, consulté le 23 septembre 2020. URL : http://journals.openedition.org/ctd/426 ; DOI : https://doi.org/10.4000/ctd.426

Ce document a été généré automatiquement le 23 septembre 2020

Communication, technologies et développement 


\title{
Frédéric Antoine (dir.), Analyser la radio. Méthodes et pratiques
}

\author{
Idé Hamani
}

\section{RÉFÉRENCE}

Frédéric Antoine (dir.), Analyser la radio. Méthodes et pratiques, De Boeck Supérieur, 2016, $256 \mathrm{p}$.

1 Analyser la radio est un ouvrage collectif proposé par les membres du Groupe de Recherches et d'Études sur la Radio (GRER). Il est dirigé par Frédéric Antoine, spécialiste reconnu de l'étude socio-économique des médias et de l'analyse des programmes. Publié en 2016 aux éditions De Boeck Supérieur, ce manuel recense des méthodes d'analyse assorties d'exemples pratiques permettant d'étudier le média radiophonique à travers plusieurs approches scientifiques, et parmi elles, les Sciences de l'information et de la communication et les Sciences du langage. Son originalité tient à l'angle méthodologique qu'il adopte et qui est inédit dans l'espace francophone, anglophone ou hispanophone, même si les études sur la radio à travers le monde sont aussi anciennes que ce média lui-même. Depuis son invention, toutes les disciplines des sciences humaines et sociales ont manifesté leur intérêt pour ce média d'accompagnement du quotidien : comme le soulignent F. Antoine et L. Gago, en tant que «moyen de communication de masse, la radio s'inscrit naturellement au cœur des sociétés, dont elle est à la fois le produit et le reflet.» (p. 35). Néanmoins, pendant une longue période, la radio est restée le «parent pauvre » de la recherche : c'est ainsi que la qualifie Geneviève Jacquinot Delaunay dans l'éditorial qui ouvre le dossier $n^{\circ} 23$ de la revue Médiamorphoses "La radio : paroles données, paroles à prendre " coordonné par Jean-Jacques Cheval en 2008. En effet, il faut attendre le début des années 2000 pour constater un regain d'intérêt pour les études scientifiques consacrées à la radio notamment avec la création des groupes de travail (IREN, ECREA, GRER). À cela s'ajoute 
l'avènement de l'internet et plus généralement des TIC qui offrent à la radio des nouvelles perspectives et suscitent un engouement scientifique au niveau mondial.

2 Analyser la radio propose bon nombre d'entrées pour comprendre les rouages du média, en étudier les acteurs, l'offre de programmes, les dispositifs, mais aussi les matériaux et les émissions diffusées. Il présente des méthodes permettant tout autant d'étudier les contenus visuels, audiovisuels et web que les publics, les usages et la méta-radio. Il vise à familiariser avec des concepts opératoires accompagnés d'exemples pratiques: le lecteur y découvre la diversité des recherches envisageables, différentes approches scientifiques et outils pédagogiques pour les apprentis chercheurs en quête de repères. Il leur fournit des réponses claires pour étudier les problématiques spécifiques au radiophonique.

3 Ce manuel est destiné particulièrement aux étudiants en fin de second cycle pour leur apporter une aide méthodologique dans la rédaction de leurs mémoires. Il s'adresse également aux doctorants qui choisissent la radiodiffusion comme objet d'étude. Enfin il représente un outil de référence pour les enseignants chercheurs de l'espace francophone.

4 L'ouvrage est structuré en huit chapitres composés de sous-chapitres. Chaque souschapitre est présenté de manière systématique avec cinq principaux points : i) « un chapeau» qui permet de se faire une idée de ce que les auteur.e.s entreprennent d'aborder; ii) une rubrique intitulée « objet de l'étude » qui se charge d'expliquer sur quoi porte la thématique proposée et qui évoque également les types d'études dont elle peut faire l'objet; iii) une rubrique « disciplines et méthodes » qui consiste à situer la thématique de recherche par rapport aux différents champs disciplinaires convoqués ainsi que les méthodes utilisées ou associées pour mener l'analyse ; iiii) un point appelé «applications » qui illustre les méthodologies et les démarches à l'aide d'exemples concrets et iiiii) une ouverture "pour aller plus loin» évoquant d'autres pistes de recherche.

5 Le premier chapitre présente les acteurs (externes et internes) de la radio. Ainsi, A. Pedroia explique que les acteurs structurels ou externes sont les personnes morales constituées sous différentes formes juridiques selon les programmes qu'elles diffusent sur un territoire. Il montre que, dans la plupart des pays, on distingue trois statuts juridiques qui régulent le marché de la radiodiffusion, notamment l'opérateur de service public, les acteurs privés à but commercial et les radios associatives ou communautaires, à but non lucratif. Tous ces acteurs sont présentés de manière que l'on comprenne aisément leurs rôles, les contenus des programmes diffusés, leurs modes de financements, les personnels qui les animent et les configurations territoriales de leur rayon de couverture. C. Deleu souligne, quant à lui, que les acteurs internes de la radio sont les professionnels qui travaillent dans l'entreprise médiatique, de la conception des programmes à la production jusqu'à la phase de diffusion.

6 Les chapitres 2 et 3 sont consacrés à la manière de mener des recherches, d'abord sur les supports de diffusion et d'écoute qui s'adaptent aux différentes périodes de l'histoire de la radio, ensuite sur le dispositif radiophonique en tant que condition matérielle de production. Dans cet ouvrage, le terme dispositif renvoie à l'infrastructure matérielle et conversationnelle. H. Glevarec souligne la variété des supports, des postes à galènes aux transistors en passant par l'autoradio et les nouveaux outils informatiques tels que l'ordinateur, les téléphones mobiles et l'internet. Pour sa part, É. Damome indique les possibilités de catégorisation et la 
typologie des radios selon leur statut juridique dans un pays donné. Il expose des méthodes pratiques pour étudier la programmation d'une station. Par exemple, selon Richard (2003) qu'il cite (p. 75), l'étude du cas français se base sur la notion de service alors que Damome $(2008,2012,2014)$ combine les statuts officiels et d'autres facteurs tels que l'offre de programmation, le public et le contenu ainsi que l'appartenance confessionnelle afin d'établir des typologies radiophoniques. Par ailleurs, H. Glevarec présente des exemples d'analyse d'interaction discursive entre un journaliste et son invité, alors que N. Becqueret montre le contrat de communication qui s'invite dans une interaction verbale, notamment lors d'un débat politique.

7 Dans le quatrième chapitre, $\mathrm{H}$. Glevarec explique comment analyser le matériau radiophonique. Il souligne par exemple que l'on peut s'intéresser aux types de musique diffusée dans une station de radio. N. Smati et L. Fauré se réfèrent à Moirand (2006) pour souligner qu'à travers les "indices de production et de réception", le chercheur peut rendre interprétables les formes linguistiques dans un discours radiophonique. Pour rendre compte de ce discours, il convient de distinguer les démarches propres à l'analyse de contenu, d'inspiration quantitative, et celles propres à l'analyse du discours. Les contributeurs précisent que «l'analyse du discours permettra plutôt d'articuler les contenus radiophoniques à la manière dont ils sont mis en forme, en scènes et en ondes. » (p. 100). Cela permet également de s'intéresser aux différents « discours narratifs » que l'on trouve à la radio.

8 A.-C. Fiévet évoque aussi la question de la langue, soit dans le cadre d'une politique linguistique, soit à propos des registres de langue utilisés par une station ; le chercheur a la possibilité de les analyser pour mettre en lumière des informations, par exemple liées à l'évolution d'une langue dans une communauté. En outre, la place de la voix dans le dispositif radiophonique et ses effets sur les auditeurs sont également des perspectives d'analyse que soulèvent N. Smati et A.-C. Fiévet (p. 117). La matérialité du son en tant que caractéristique de la radio peut être une source d'inspiration particulière car, selon Chion (1998: 23), le son "est une onde qui, à la suite de l'ébranlement d'une ou plusieurs sources nommées corps sonores, se propage [...] et, au passage, touche ce qu'on appelle l'oreille, où elle donne matière à des sensations auditives [...]» (Deleu et Glevarec, p. 122). Les contributeurs proposent des outils méthodologiques prenant en compte tous les aspects du son radiophonique. D'autre part, L. Gago suggère au chercheur de s'intéresser aux nouveaux canaux de diffusion comme l'internet qui, depuis son apparition, constitue un objet d'étude prometteur. Ce dispositif permet de donner la parole aux auditeurs internautes qui s'engagent dans le débat démocratique en réagissant directement sur les pages des réseaux sociaux des stations (pp. 129-130).

9 Les chapitres 5 et 6 sont consacrés aux méthodes pour analyser les émissions radiophoniques en termes de genre, de conditions de production et de contenus diffusés ainsi que de contenus visuels et audiovisuels. Pour ce faire, l'identification de la catégorie de programmes à laquelle appartient une émission s'avère nécessaire. Les genres radiophoniques les plus connus sont entre autres les « émissions d'information, émissions de débat, émissions de fiction, émissions de libre antenne, émissions musicales, jeux, etc. » (Deleu et Equoy Hutin, p. 134). Mais, il est important de savoir que certains genres sont composés des sous-genres. Ainsi, analyser les émissions en termes de genre exige une méthodologie particulière. Le chercheur non averti peut confondre les classifications puisque les professionnels et les chercheurs n'adoptent 
parfois pas les mêmes. C. Deleu et S. Equoy-Hutin signalent que "la relation d'appartenance d'une émission à un genre n'est donc pas biologique. Par conséquent, le chercheur doit faire preuve d'une certaine prudence et d'une certaine distance pour définir un genre et classer les émissions dans telle ou telle catégorie, même dans les situations où un consensus, tant en termes de conditions de production que de réception, semble s'imposer.» (p. 136). Pour catégoriser le genre, on peut donc s'appuyer sur l'histoire de la radio, établir une comparaison et suivre son évolution.

Quel que soit le genre radiophonique que l'on envisage d'analyser, il est important de comprendre les conditions dans lesquelles est produit le programme et de relever les éléments qui ont concouru à sa réalisation. Dans le contrat de communication et d'information médiatique de Charaudeau (2011), C. Deleu et S. Equoy-Hutin relèvent qu'« on ne peut pas analyser la radio sans comprendre quels sont les paramètres et les processus qui influent et orientent la production médiatique, quel que soit le type d'émission ou le genre auquel elle se rattache. » (p. 141). De plus, étudier les contenus des radios suppose de prendre en compte deux types d'outils d'analyse: analyse qualitative et quantitative. Les règles et les procédures de ces analyses sont explicitement détaillées par É. Damome et S. Equoy-Hutin de sorte que le chercheur procède directement à leur application sur son propre corpus.

11 Par ailleurs, l'avènement de l'internet permet aux radios de développer des espaces d'information autres que le système d'écoute traditionnel. L'analyse des sites web radio et de leur architecture permet d'interpréter la sémantique des mots et des images utilisés. Dès lors, le chercheur peut travailler sur ce que L. Fauré et L. Gago appellent «radio visuelle» et «radio filmée» (p. 162) permettant aux auditeurs de voir l'intérieur des studios et les équipes de travail. Cette nouvelle façon de concevoir et d'étudier la radio est prometteuse, dans le sens où elle permet de mesurer l'évolution de la radio en fonction du développement technologique dans la société, et d'initier des études de terrain.

Les chapitres 7 et 8 analysent les publics, les usages et les discours des médias dans lesquels on parle de la radio. En radiodiffusion tout comme dans les autres médias, connaître les publics, leurs goûts et leurs temps d'écoute est nécessaire pour l'image de la station et de son bon fonctionnement. C'est pourquoi de nombreuses études d'audience sont réalisées par les stations, mais aussi parfois par des instituts de mesure d'audience, utilisant des méthodes d'enquête quantitative et souvent qualitative. En guise d'exemple, F. Antoine explique qu' « en France, Médiamétrie mesure l'audience de la radio en menant chaque année 126000 interviews téléphoniques réparties sur dix mois auprès d'un échantillon de la population âgé de 13 ans et plus [...], alors qu'en Suisse, l'opérateur Mediapulse mesure l'audience radio via un audiomètre portable ayant la forme d'un bracelet-montre que les panélistes doivent porter 24/24 pendant une semaine, puis retourner au laboratoire d'analyse des données. » (p. 172).

13 L'ouvrage propose également aux chercheurs de s'intéresser aux effets de la radio sur les auditeurs. Ce média, comme d'autres, peut être un outil mis au service d'une finalité d'information, de distraction, voire de propagande politique. Mais la radio peut aussi être un "contre-pouvoir» qui sensibilise les auditeurs à leurs droits et les incite à participer pleinement au bon fonctionnement de la démocratie. La question du rapport entre le public et la radio nécessite de nouvelles analyses prenant en compte la participation des auditeurs sur les réseaux sociaux. 
14 Étudier le discours sur la radio, enfin, apporte de nouvelles informations. Il s'agit de voir comment les autres médias en l'occurrence la presse écrite, la télévision, mais aussi le cinéma et l'internet parlent du média radiophonique. La littérature, les expositions et les festivals consacrés à la radio, constituent également un autre angle d'étude et ouvrent des pistes d'analyse qui peuvent susciter des intérêts particuliers en recherche.

Analyser la radio est le premier ouvrage collectif francophone qui propose des entrées à la fois théoriques et pratiques pour étudier la radiophonie. Ce manuel, unique en son genre, traite des thématiques de recherche sur la radio au travers des matériaux, des émissions et des contenus, mais aussi des publics et des métadiscours. Il privilégie également une démarche active et la mise en pratique pour analyser le média radio, ses acteurs et ses dispositifs de diffusion. Il sera d'une grande utilité pour tous ceux qui s'intéressent à la radio et qui veulent comprendre son fonctionnement en tant qu'acteur social.

\section{AUTEURS}

\section{IDÉ HAMANI}

Université de Bourgogne Franche-Comté, ELLIADD-CCM 